\title{
Donación después de muerte cardiovascular en Chile
}

\author{
Donation after cardiovascular death in Chile
}

Pablo Pérez Castro ${ }^{1,2}$, Raimundo Santolaya Cohen ${ }^{1,3}$ y José Palacios Junemann ${ }^{1,2}$

Chile es un país de 17.574.003 personas ${ }^{1}$ y durante el año 2020 hubo solo 141 donantes efectivos acorde al Sistema Integrado de Donación y Trasplante $^{2}$. El año anterior, la tasa nacional de donantes fue de 10,4 por millón de habitantes, posicionándonos lejos de países vecinos como Uruguay $(22,86)$, Argentina $(19,6)$ y Brasil (18); y a un abismo de países líderes en donación de órganos como España (49.61) o Estados Unidos $(36,88)^{3}$.

Para fomentar la donación de órganos se han realizado campañas comunicacionales ${ }^{4}$, se adoptó un modelo de inscripción automática en registro de donantes (sistema opt-out) ${ }^{5}$ y se ha promovido el uso de donantes vivos ${ }^{6,7}$. Una deuda pendiente es intentar maximizar aún más los tipos de donantes de órganos de origen cadavérico.

Una forma novedosa de aumentar el pool de donantes cadavéricos es incluir la donación después de muerte cardiovascular (DCD) como una categoría de donante adicional a la muerte cerebral ${ }^{8}$. Este grupo de donantes son pacientes con pronóstico ominoso en los que se han agotado posibilidades terapéuticas y se encuentran próximos a fallecer, pero que no cumplen criterios neurológicos de muerte cerebral.

El proceso (al igual que la donación por muerte cerebral), requiere el consentimiento de los familiares. Una vez consentido el donante y declarada la muerte cardiovascular por un equipo médico imparcial (requiere 5 minutos sin evidencia de circulación), un equipo quirúrgico procede con la procuración de órganos. Esta técnica ha sido ampliamente practicada a nivel mundial desde hace veinte años ${ }^{9}$, y se realiza bajo estrictos estándares éticos avalados por el Institute of Medicine $e^{10}$, el American College of Critical Care Medicine ${ }^{11}$ y la Society of Critical Care Medicine ${ }^{12}$.

El uso de órganos procedentes de donantes DCD ha demostrado equivalencia a largo plazo en comparación a donantes por muerte cerebral en órganos como riñón, hígado, páncreas y pulmón ${ }^{12-15}$. Además, la técnica permite la donación de tejidos y córneas. Mediante la incorporación de donantes DCD tenemos la potencialidad de aumentar el número de donantes en Chile en más menos $20 \%{ }^{16}$.

En un contexto nacional donde la mortalidad en lista de espera es de $17 \%$ en el caso de pacientes esperando un pulmón y de un alarmante $32 \%$ en caso de pacientes esperando un hígado ${ }^{17}$, se hace prioritario agotar las posibilidades de ampliar los tipos de donantes.

Introducir este tipo de donación de órganos en nuestro país es uno de los pasos más importantes que podemos hacer para ofrecer a nuestra población la posibilidad de vivir más y mejor.

\section{Bibliografía}

1. Censos de Población y Vivienda [Internet]. Default. [citado el 11 de enero de 2021]. Available from: http://www. ine.cl/estadisticas/sociales/censos-depoblacion-y-vivienda.

2. Yo Dono Vida: Ministerio de Salud [Internet]. [citado el 11 de enero de 2021]. Available from: https://yodonovida. minsal.cl/statistics/public/show/25.

3. Newsletter Dec 2020 pdf [Internet]. [citado el 11 de enero de 2021]. Available from: https://www.irodat.org/img/ database/pdf/Newsletter $\% 20 \mathrm{Dec} \% 20$ 2020\%20.pdf.

4. Minsal lanza campaña de donación de órganos "El mejor regalo de tu vida" [Internet]. Ministerio de Salud-Gobierno de Chile. [citado el 11 de enero de 2021]. Available from: https://www.minsal.cl/ minsal-lanza-campana-de-donacion-deorganos-el-mejor-regalo-de-tu-vida/

5. Chile B del CN de Ley Fácil [Internet]. Biblioteca del Congreso Nacional de Chile; 2014 [citado el 11 de enero de
'Unidad de Trasplante, Clínica Alemana de Santiago. Santiago, Chile. 2Departamento de Cirugía Occidente, Hospital San Juan de Dios, Universidad de Chile. Santiago, Chile. 3epartamento de Cirugía Oriente, Instituto Nacional de Tórax, Universidad de Chile.

Santiago, Chile.

Recepción 2021-01-23 aceptado 2021-01-24

Correspondencia a: Dr. Pablo Perez Castro dr.pabloperezc@gmail.com
2021]. Available from: https://www.ben. cl/leyfacil.

6. Aguiló MJ, Matus FC, Leiva LL, Pérez CP, Castillo HF, Vergara MJ, et al. Nefrectomía laparoscópica de donante vivo: experiencia de 75 casos consecutivos. Rev Chil Cir. [Internet]. 2015 [citado el 12 de enero de 2021];67(1):57-60. Available from: https://scielo.conicyt.cl/scielo. php?script $=$ sci_abstract\&pid $=$ S0718$40262015000100009 \& \operatorname{lng}=\mathrm{es} \& \mathrm{nrm}=\mathrm{iso} \&$ tlng=es. 
EDITORIAL

7. www.ilogica.cl I. Trasplante de hígado con donante vivo en adultos: una posibilidad real [Internet]. Escuela de Medicina. [citado el 12 de enero de 2021]. Available from: https://medicina.uc.cl/noticias/ trasplante-higado-donante-vivo-adultospodria-salvarle-la-vida-cientos-personas/

8. Thuong M, Ruiz A, Evrard P, Kuiper M, Boffa C, Akhtar MZ, et al. New classification of donation after circulatory death donors definitions and terminology. Transplant International [Internet]. 2016 [citado el 12 de enero de 2021];29(7):74959. Available from: https://onlinelibrary. wiley.com/doi/abs/10.1111/tri.12776

9. Love RV. First successful lung transplantation using a nonheartbeating donor. J Heart Lung transplant 1005;14:88. Google Scholar [Internet]. [citado el 12 de enero de 2021]. Available from: https://scholar.google.com/ scholar_lookup?title=Successful $\% 20$ lung $\% 20$ transplantation $\% 20$ using $\% 20 \mathrm{a} \% 20$ non-heart-beating $\% 20$ donor\&journal $=\mathrm{J} \% 20$ Heart $\% 20$ Lung $\% 20$ transplant\&volume $=14 \&$ publication_year $=1995 \&$ author $=$ Love $\% 2 C R$. B.\&author $=\mathrm{St}$ ringham $\% 2$ CJ.C.\&author $=$ Chomiak $\% 2 \mathrm{CP}$ .N.

10. Medicine I of. Non-Heart-Beating Organ Transplantation: Practice and Protocols [Internet]. 1999 [citado el 12 de enero de 2021]. Available from: https://www. nap.edu/catalog/9700/non-heart-beatingorgan-transplantation-practice-andprotocols.

11. Ethics Committee, American College of Critical Care Medicine, Society of Critical Care Medicine. Recommendations for nonheartbeating organ donation. A position paper by the Ethics Committee, American College of Critical Care Medicine, Society of Critical Care Medicine. Crit Care Med. 2001;29:1826-31.

12. Schaapherder A, Wijermars LGM, de Vries DK, de Vries APJ, Bemelman FJ, van de Wetering J, et al. Equivalent Long-term Transplantation Outcomes for Kidneys Donated After Brain Death and Cardiac Death: Conclusions From a Nationwide Evaluation. EClinicalMedicine [Internet]. 2018 [citado el 12 de enero de 2021];4:25-31. Available from: https://www.thelancet.com/journals/ eclinm/article/PIIS2589-5370(18)300324/abstract

13. Scalea JR, Redfield RR, Foley DP. Liver transplant outcomes using ideal donation after circulatory death livers are superior to using older donation after brain death donor livers. Liver Transpl. 2016;22:1197204.

14. Leemkuil M, Leuvenink HGD, Pol RA. Pancreas Transplantation from Donors after Circulatory Death: an Irrational Reluctance? Curr Diab Rep [Internet]. 2019 Nov 18 [citado el 12 de enero de 2021];19(11):129. Available from: https://doi.org/10.1007/s11892-0191238 -y.

15. Raemdonck DEV, Keshavjee S, Levvey B, Cherikh WS, Snell G, Erasmus ME, et al. 5-Year Results from the ISHLT DCD Lung Transplant Registry Confirm Excellent Recipient Survival from Donation after Circulatory Death Donors. The Journal of Heart and Lung Transplantation [Internet]. 2019 [citado el 12 de enero de 2021];38(4):S103. Available from: https://www.jhltonline. org/article/S1053-2498(19)30242-6/ abstract.

16. Expanding the organ donor pool [Internet]. [citado el 12 de enero de 2021]. Available from: https://www.uhn.ca:443/ corporate/News/Pages/expanding_organ_ donor_pool.aspx.

17. Arriagada AM, Maquilón S, Benítez C. Donación y trasplante de órganos en Chile. Rev Med Chile [Internet]. 2018 [citado el 11 de enero de 2021];146(5):675-6. Available from: https://scielo.conicyt.cl/scielo. php?script $=$ sci_abstract\&pid=S0034 $98872018000500675 \& \operatorname{lng}=\mathrm{es} \& \mathrm{nrm}=\mathrm{iso} \&$ tlng=es 